\title{
High-Energy Sub-Optical-Cycle Parametric Waveform Synthesizer
}

\author{
Giovanni Cirmi, Giulio M. Rossi, Shaobo Fang, Shih-Hsuan Chia, \\ Oliver D. Mücke, Cristian Manzoni, Paolo Farinello, Giulio Cerullo \\ and Franz X. Kärtner
}

\begin{abstract}
We compressed and characterized using FROG a 3-channel, multi-mJ, sub-optical-cycle parametric waveform synthesizer in the visible and infrared $(0.5-$ $2.2 \mu \mathrm{m})$. The synthesizer will become a versatile tool for strong-field physics experiments.
\end{abstract}

Waveform Nonlinear Optics aims to study and control the nonlinear interaction of matter with extremely short optical waveforms custom-tailored within an optical cycle of light. Several new applications arise in attoscience and strong-field physics, e.g., the generation of intense isolated attosecond XUV pulses [1], relativistic laserplasma interactions and laser-driven electron acceleration [2], launching valenceelectron wavepacket dynamics in atoms and molecules [3], and control of sub-cycle electron transport in solids [4].

Different technological approaches [5] have recently been pursued to generate such waveforms. Waveform synthesis based on supercontinuum generation in hollow-core fiber compressors represents a rather mature technology and has led to the generation of sub-cycle optical pulses (covering 260-1,100 nm) [3], however,

G. Cirmi ( $\square)$ - G.M. Rossi · S. Fang · S.-H. Chia · O.D. Mücke · F.X. Kärtner Center for Free-Electron Laser Science, Deutsches Elektronen-Synchrotron DESY, Notkestraße 85, 22607 Hamburg, Germany

e-mail: giovanni.cirmi@cfel.de

F.X. Kärtner

Physics Department, University of Hamburg, Luruper Chaussee 149, 22761 Hamburg, Germany

G. Cirmi · G.M. Rossi · S. Fang · S.-H. Chia · O.D. Mücke · F.X. Kärtner

The Hamburg Center for Ultrafast Imaging, Luruper Chaussee 149, 22761 Hamburg, Germany

F.X. Kärtner

Department of Electrical Engineering and Computer Science and Research Laboratory of Electronics, Massachusetts Institute of Technology, Cambridge, MA 02139, USA

C. Manzoni $\cdot$ P. Farinello $\cdot$ G. Cerullo

IFN-CNR Dipartimento di Fisica, Politecnico di Milano, Piazza Leonardo da Vinci 32, 20133 Milan, Italy

(C) Springer International Publishing Switzerland 2015

K. Yamanouchi et al. (eds.), Ultrafast Phenomena XIX,

Springer Proceedings in Physics 162, DOI 10.1007/978-3-319-13242-6_192 
the energy throughput is typically limited to several tens or hundreds of $\mu \mathrm{Js}$ (depending on bandwidth) due to ionization in the gas medium. Alternatively, parametric waveform synthesizers [6-8] feature straightforward energy scalability $[1,2]$ and can also be extended into the important mid-IR region for the realization of bright coherent tabletop high-harmonic sources in the water-window and keV X-ray region [9]. Parametric waveform synthesizers can be classified into (i) sequential and (ii) parallel schemes. In the sequential approach [2, 8], different spectral regions are amplified in subsequent amplification stages employing different phase-matching conditions without ever splitting the beam. The advantage of this sequential approach is the avoidance of synchronization issues, which comes at the expense of the grand challenge to compress the entire bandwidth with the same dispersive elements at once. In contrast, parallel synthesis schemes [3, 6, 7] offer much greater flexibility by splitting the bandwidth into separate channels, in which the different spectral regions can individually be amplified and/or compressed. Importantly, the coherent synthesis of reproducible waveforms in parallel schemes requires sub-cycle timing synchronization as well as tight stabilization of the relative phases among the different channels, and carrier-envelope phase (CEP) stability.

In 2013, we proposed and already reported first amplification results (see Fig. 1) for a 3-channel multi-mJ sub-cycle parametric waveform synthesizer [7]. Our synthesizer is driven by a non-CEP-stable cryogenically cooled Ti:sapphire amplifier (150 fs, $18 \mathrm{~mJ}, 1 \mathrm{kHz})$. We generate a CEP-stable seed continuum $(0.5-2.5 \mu \mathrm{m})$ by white-light generation in a YAG crystal pumped by the second harmonic $(1.06 \mu \mathrm{m})$ of the CEP-stable idler of a NIR OPA. The continuum is then split with dichroic mirrors and seeds three BBO-based OPA channels: a VIS non-collinear OPA (NOPA), a NIR and an IR degenerate OPA (DOPA), pumped by the pulses at $0.8 \mu \mathrm{m}$ (IR DOPA) and by its second harmonic at $0.4 \mu \mathrm{m}$ (VIS NOPA, NIR DOPA).

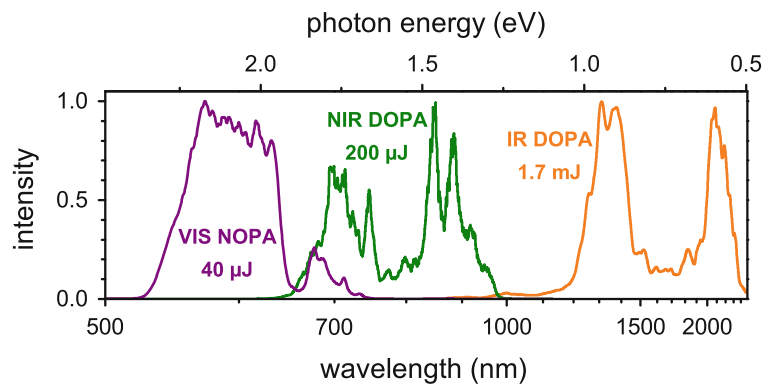

Fig. 1 Output spectra so far obtained from the 2-octave-wide multi-mJ parametric synthesizer: second-stage output spectrum of the VIS NOPA (violet), third-stage spectra of the NIR DOPA (green) and IR DOPA (orange). None of these spectra is contaminated by superfluorescence backgrounds. The spectra (VIS NOPA/NIR DOPA/IR DOPA) support 6.7/5.2/5.2 fs pulses corresponding to $3.4 / 2.1 / 1.1$ optical cycles at $595 / 750 / 1390 \mathrm{~nm}$ center wavelengths. The transform-limited (TL) pulse duration from the synthesis of these three spectra (assuming 1:1.3:1 intensity weighting and all CEPs to be 0) is 1.9 fs FWHM (not shown), corresponding to 0.7 optical cycles at $785 \mathrm{~nm}$ center wavelength 
Figure 1 shows the output spectra and pulse energies obtained so far from the three OPA channels. After two amplification stages only, all channels yield $\sim 40-80 \mu \mathrm{J}$ each.

Here, we present first FROG pulse-characterization results of all three spectral channels, up to the second stage for the VIS NOPA and for the NIR DOPA, and up to the third stage for the IR DOPA. The FROG measurement of the last IR DOPA stage, not shown here, yields a 9.9-fs pulse duration. These results demonstrate the feasibility to recompress all three channels simultaneously close to the Fourier limit and show the flexibility of our dispersion compensation scheme for different experimental situations. The compressed third stage of the IR DOPA is currently delivering $0.3 \mathrm{~mJ}$ energy per pulse, but we have already demonstrated the energy scalability up to $1.7 \mathrm{~mJ}$ (superfluorescence free) as shown in Fig. 1. We emphasize that the FROG results shown in Fig. 2 were measured at the actual waveform synthesis point, i.e., each of the three pulses propagates through its complete synthesizer channel and the beam combiner and thus experiences the correct dispersion and nonlinearity on its way. In other words, the synthesis can later directly be performed from these pulses after locking their relative timing using feedback loops with balanced optical cross-correlators, that can achieve sub-cycle synchronization with $<30$-as RMS timing jitter [6]. The dispersion compensation scheme [10] includes custom-designed double-chirped mirror (DCM) pairs and dichroic beam splitters/combiners, plates and wedges $\left(\mathrm{SiO}_{2}, \mathrm{ZnSe}\right)$ for dispersion fine-tuning, and the $\mathrm{CaF}_{2}$ window of our future experiment's vacuum chamber.
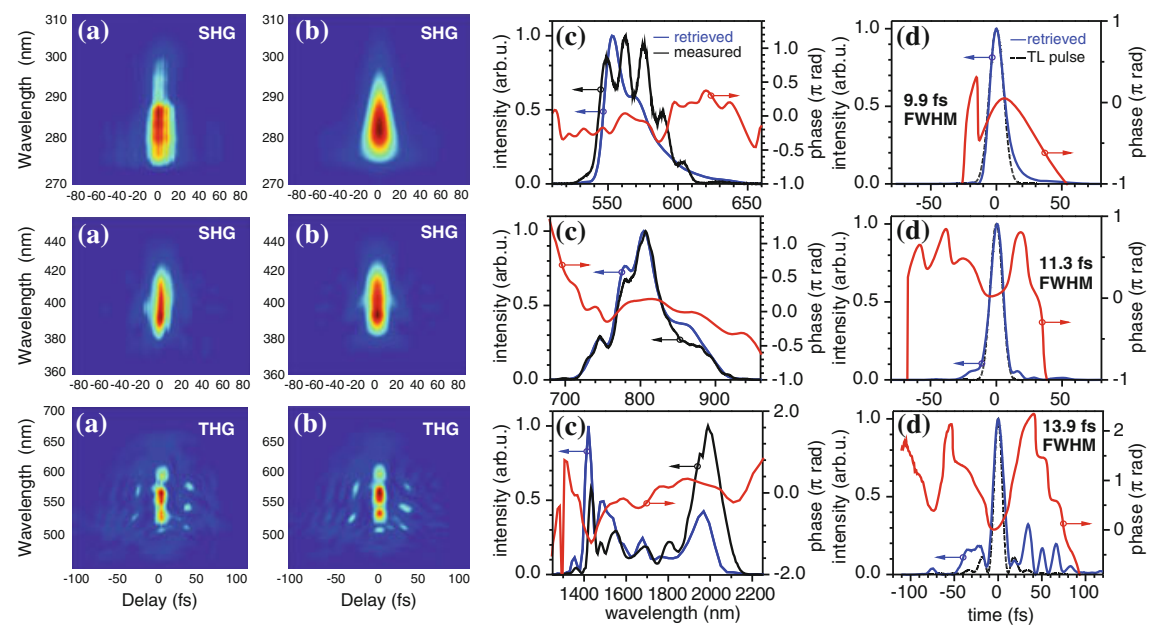

Fig. 2 FROG characterization of the second-stage OPA outputs from the three channels at the synthesis point. The VIS NOPA (top row) and NIR DOPA (middle row) are characterized by means of SHG-FROG, the IR DOPA (bottom row) using surface THG-FROG. a Measured and b retrieved FROG traces. c Measured spectrum, retrieved spectral intensity and phase. d Retrieved temporal intensity and phase profiles as well as TL intensity profile. The retrieved FWHM pulse durations are indicated. No marginal correction was applied to the THG-FROG trace in the bottom row 
The next step will be the waveform synthesis from all three channels at the $\mathrm{mJ}$ level. Synthesized waveforms (not shown here) as short as 2.7 fs FWHM can be obtained from the second OPA stages for different relative phases, computed from the measured spectral amplitudes and phases in Fig. 2c.

Our parametric waveform synthesizer spanning more than two octaves already provides unprecedented opportunities for strong-field experiments on solids [4] and nanostructured solid-state devices [11]. After compressing the full 3-channel 3 -stage system [7], it will become a versatile tool for controlling strong-field interactions in atoms and molecules and for attosecond pump-probe spectroscopy using VIS/IR and XUV/soft-X-ray pulses.

\section{References}

1. E. J. Takahashi et al., Nat. Commun. 4, 2691 (2013); E. J. Takahashi et al., Phys. Rev. Lett. 104, 233901 (2010).

2. L. Veisz et al., CLEO Pacific Rim 2013, TuD2-3.

3. A. Wirth et al., Science 334, 195 (2011). Also see: T. T. Luu et al., QELS 2013, QF1C.6.

4. O. D. Mücke, Phys. Rev. B 84, 081202(R) (2011); S. Ghimire et al., Nature Phys. 7, 138 (2011); A. Schiffrin et al., Nature 493, 70 (2013); M. Schultze et al., Nature 493, 75 (2013).

5. C. Manzoni et al., Laser Photonics Rev., DOI 10.1002/lpor.201400181 (2014).

6. S.-W. Huang et al., Nature Photonics 5, 475 (2011); C. Manzoni et al., Opt. Lett. 37, 1880 (2012).

7. G. Cirmi et al., UFO IX, 2013, We3.3; O. D. Mücke et al., CLEO 2013, CTh3H.3; S. Fang et al., CLEO Pacific Rim 2013, WB3-1; G. M. Rossi et al., CLEO 2014, SF1E.3.

8. A. Harth et al., Opt. Express 20, 3076 (2012).

9. E. J. Takahashi et al., Phys. Rev. Lett. 101, 253901 (2008); T. Popmintchev et al., Science 336, 1287 (2012).

10. S.-H. Chia et al., Optica 1, 315 (2014); S.-H. Chia et al., patent EP14155053, "Chirped dichroic mirror and a source for broadband light pulses."

11. P. D. Keathley et al., Ann. Phys. 525, 144 (2013); H. Ye et al., Ultrafast Phenomena 2014, Chapter no. 163. 